\title{
AMCoR
}

Asahikawa Medical University Repository http://amcor.asahikawa-med.ac.jp/

International journal of hematology. (2016.8) 104(2):175-81.

In vivo behavior of NTBI revealed by automated quantification system.

Satoshi Ito, Katsuya Ikuta, Daisuke Kato, Addo Lynda, Kotoe Shibusa, Noriyasu Niizeki, Yasumichi Toki, Mayumi Hatayama, Masayo Yamamoto, Motohiro Shindo, Naomi Iizuka, Yutaka Kohgo, Mikihiro Fujiya 
4 Satoshi Ito ${ }^{\mathrm{a}, \mathrm{b}}$, Katsuya Ikuta ${ }^{\mathrm{b}}$, Daisuke Kato ${ }^{\mathrm{c}}$, Addo Lynda ${ }^{\mathrm{b}}$, Kotoe

5 Shibusa $^{\mathrm{b}}$, Noriyasu Niizeki ${ }^{\mathrm{d}}$, Yasumichi Toki ${ }^{\mathrm{b}}$, Mayumi Hatayama ${ }^{\mathrm{b}}$,

6 Masayo Yamamoto ${ }^{\mathrm{b}}$, Motohiro Shindo ${ }^{\mathrm{b}}$, Naomi Iizukac, Yutaka Kohgo ${ }^{\mathrm{b}, \mathrm{e}}$,

$7 \quad$ Mikihiro Fujiyab

8

9

10

11

12

13

\section{In vivo behavior of NTBI revealed by automated quantification system}

8

a Department of Oncology for Local Community Cooperation, Asahikawa Medical University Hospital, Asahikawa, Japan

${ }^{\mathrm{b}}$ Division of Gastroenterology and Hematology/Oncology, Department of Medicine, Asahikawa Medical

University, Asahikawa, Hokkaido, Japan

${ }^{\mathrm{c}}$ Research and Development Department, Shino-Test Corporation, Sagamihara, Kanagawa, Japan

${ }^{\mathrm{d}}$ Department of Medical Laboratory and Blood Center, Asahikawa Medical University Hospital,

Asahikawa, Hokkaido, Japan

e Department of Gastrointestinal Immunology and Regenerative Medicine, Asahikawa Medical University, 2-1-1-1, Midorigaoka-Higashi, Asahikawa, Hokkaido 078-8510, Japan. 
2 Contact information for correspondence:

3 Katsuya Ikuta, M.D., Ph.D.

$4 \quad$ Assistant Professor

5 Division of Gastroenterology and Hematology/Oncology

6 Department of Medicine, Asahikawa Medical University

7 2-1-1-1 Midorigaoka-Higashi, Asahikawa, Hokkaido 078-8510, Japan

$8 \quad$ Tel: $\quad+81-166-68-2462$

$9 \quad$ Fax: $\quad+81-166-68-2469$

10 E-mail: ikuta@asahikawa-med.ac.jp 


\section{Abstract}

2 Non-Tf-bound iron (NTBI), which appears in serum in iron overload, is thought to contribute to organ

3 damage; the monitoring of serum NTBI levels may therefore be clinically useful in iron overloaded

4 patients. However, NTBI quantification methods remain complex, limiting their use in clinical practice.

5 To overcome the technical difficulties often encountered, we recently developed a novel automated NTBI

6 quantification system capable of measuring large numbers of samples. In the present study, we

7 investigated the in vivo behavior of NTBI in human and animal serum using this newly established

8 automated system. Average NTBI in healthy volunteers was $0.44 \pm 0.076 \mu \mathrm{M}$ (median: $0.45 \mu \mathrm{M}$, range:

$9 \quad 0.28-0.66 \mu \mathrm{M}$ ), with no significant difference between sexes. Additionally, serum NTBI rapidly

10 increased after iron loading, followed by a sudden disappearance. NTBI levels also decreased in

11 inflammation. The results indicate that NTBI is a unique marker of iron metabolism, unlike other markers

12 of iron metabolism, such as serum ferritin. Our new automated NTBI quantification method may help to

13 reveal the clinical significance of NTBI and contribute to our understanding of iron overload.

14 


\section{Key words:}

2 non-transferrin bound iron (NTBI), automated quantification system, iron overload, inflammation

3

4

5

6

7

8

9

10

11

12

13

14

15

16

17

18 
Iron is an essential metal in the body, but in excess, accumulates in body organs, causing organ

3 damage through free radical production. Systemic iron homeostasis is therefore usually tightly regulated,

4 however in conditions such as prolonged transfusion, iron overload may occur [1, 2].

5 Iron absorbed into the gastrointestinal tract is transported in the blood stream bound to

6 transferrin (Tf), whereby approximately 30\% of Tf-binding sites are saturated with iron. However,

7 Tf-binding sites become fully saturated in iron overload, and free unbound iron called non-Tf-bound iron

8 (NTBI), appears in serum. Iron overload damages various body organs, and because NTBI, which is

9 easily absorbed, is thought to contribute to those damages, monitoring serum NTBI levels may be crucial

10 in assessing clinical markers in patients with iron overload [3].

11 Recent studies demonstrate that serum NTBI or its redox active fraction, labile plasma iron

12 (LPI), is elevated in pathological conditions such as in end stage renal disease patients on hemodialysis,

13 patients with myelodysplastic syndrome (MDS) receiving transfusion, acute leukemia patients receiving

14 chemotherapy, patients after hematopoietic cell transplantation and hereditary hemochromatosis patients

15 [4-10], demonstrating that NTBI likely contributes to disease progression in these patients. Measuring

16 serum NTBI may therefore be useful in evaluating, and monitoring the risk of iron toxicity in patients

17 [11]. In most of the above-mentioned studies however, NTBI measurement was only carried out in a

18 limited number of specimens, mainly because of the expensive and complex nature of NTBI

19 quantification methods, and explains why to date, no documented reports of NTBI evaluation in a larger

20 patient population are available.

21 To overcome the technical difficulties often encountered, and to make the NTBI quantification

22 process more convenient, we established a novel automated NTBI quantification method that will be

23 useful in diagnostic testing in clinical laboratories [12]. This method employs a completely automated

24 system that is also capable of measuring a large number of samples. We confirmed that this system is 
1 fully compatible with previously established methods, such as high-performance liquid chromatography

2 and atomic absorption spectroscopy-based assays. In the present study, we investigated the in vivo

3 behavior of NTBI using our automated quantification system to introduce the method to clinical practice. 4 


\section{Materials and Methods}

\section{Human serum samples}

Human serum samples, obtained from 41 healthy volunteers and 116 patients receiving treatment

at the Asahikawa Medical University, were collected from November 2012 to January 2014. The study

5 protocol was approved by the Ethical Committee of the Asahikawa Medical University, and all study subjects provided informed consent. Average age of the healthy volunteers was 34.4 years (range, 20-58

7 years), which included 16 males and 25 females. Average age of the patients was 60.8 years (range,

8 16-86 years), which included 60 males and 56 females. Diagnosis of the 116 patients showed malignant

9 lymphoma (ML; n=34), acute myeloid leukemia (AML; n=11), myelodysplastic syndromes (MDS;

$10 \mathrm{n}=10$ ), multiple myeloma (MM; $\mathrm{n}=10$ ), malignant tumors in digestive organs $(\mathrm{n}=15)$, chronic myeloid

11 leukemia (CML; n=7), myeloproliferative neoplasms (MPN; $n=4$ ), Crohn's disease (CD; $n=3$ ) and

12 others $(\mathrm{n}=22)$. The other group included iron deficiency anemia $(\mathrm{n}=2)$, idiopathic thrombocytopenic

13 purpura $(\mathrm{n}=2)$, pure red cell aplasia $(\mathrm{n}=2)$, liver cirrhosis $(\mathrm{n}=2)$, iron overload $(\mathrm{n}=2)$, non-alcoholic

14 steatohepatitis $(\mathrm{n}=1)$, liver abscess $(\mathrm{n}=1)$, paroxysmal nocturnal hemoglobinuria $(\mathrm{n}=1)$, rheumatoid

15 arthritis $(\mathrm{n}=1)$, Hodgkin lymphoma $(\mathrm{n}=1)$, hemophagocytic syndrome $(\mathrm{n}=1)$, secondary polycythemia

$16(\mathrm{n}=1)$, donor for hematopoietic stem cell transplantation $(\mathrm{n}=1)$, macroglobulinemia $(\mathrm{n}=1)$, acute

17 lymphocytic leukemia $(n=1)$, neuroendocrine tumor $(n=1)$, and cancer of unknown primary $(n=1)$. Of

18 the 116 patients, 14 received either autologous or allogeneic hematopoietic stem cells transplantations. 
1 creatinine, C-reactive protein (CRP), albumin and serum ferritin levels in the healthy volunteers were

2 determined, and in the patient subjects, data from patient records were utilized (Table 1). Serum iron

3 (sFe) and unsaturated iron binding capacity (UIBC) were quantified using commercial kits (QuickAuto

$4 \quad$ Neo Fe and QuickAuto Neo UIBC, Shino-test, Tokyo, Japan) and transferrin saturation (\%) was

5 calculated using the following formula: sFe / $(\mathrm{sFe}+\mathrm{UIBC}) \times 100$. The serum samples were kept frozen

6 at $-20^{\circ} \mathrm{C}$ until NTBI quantification. The frozen samples were thawed at $37^{\circ} \mathrm{C}$ until no solid particles

7 were visible, and then chilled on ice.

\section{Determination of NTBI in mice and rat serum} 20-week-old C57BL/6 male mice (Charles River Laboratories Japan, Yokohama, Japan) were randomly placed into 3 groups (control, Fe $5 \mathrm{mg}$, Fe $50 \mathrm{mg}$ ) with each group composed of 5 mice.

11 Physiological saline, $1 \mathrm{mg} /$ head/day of iron-dextran (Sigma-Aldrich, St. Louis, MO) and $10 \mathrm{mg} / \mathrm{head} / \mathrm{day}$

12 of iron-dextran were intraperitoneally injected into the mice in the respective study groups for 5 days.

13 Serum samples were collected from the mice 2 days after the last treatment. 17-week-old Wister rats (Charles River Laboratories Japan, Yokohama, Japan) were randomly placed into 2 groups (control and iron sucrose), with each group composed of 3 rats. Serum samples were

16 collected before and after they received intravenous injections of physiological saline and iron sucrose

17 (Fesin; Nichiiko, Tokyo, Japan) in $10 \mathrm{mg} / \mathrm{kg}$ doses; Serum samples were collected from each rat before and at 1,3 and 6 hours after injections. 
placed into 4 groups (control, iron, lipopolysaccharide (LPS), iron + LPS) with 3 animals in each group.

3 Physiological saline and iron citrate (Ferromia; Eisai, Tokyo, Japan) were orally administered in doses of

$42 \mathrm{mg} / \mathrm{kg}$ to the mice in the control and iron groups respectively. To evaluate the effect of inflammation on

5 serum NTBI concentration, $2.5 \mathrm{mg} / \mathrm{kg}$ of LPS (E. coli 026; Wako, Osaka, Japan) was intraperitoneally

6 injected into the mice in the LPS group. The LPS-treated rats were further administered with iron citrate

7 as described above (iron + LPS group). Serum samples were collected from each rat before and at 1, 3

8 and 6 hours after treatment.

All experimental procedures were approved by the animal experiments committee of the

10 Asahikawa Medical University (Hokkaido, Japan) based on guidelines for the protection of animals.

\section{Statistical Analysis}

14 test was used. Student paired t-test was performed for normal distribution variables. $\mathrm{r}>0.4$ was 


\section{Results}

\section{NTBI in healthy volunteers and patients}

Average NTBI in healthy volunteers was $0.44 \pm 0.076 \mu \mathrm{M}$ (median: $0.45 \mu \mathrm{M}$, range: $0.28-0.66$

$\mu \mathrm{M}) ; 0.45 \pm 0.078 \mu \mathrm{M}$ in males $(\mathrm{n}=16)$ and $0.43 \pm 0.075 \mu \mathrm{M}$ in females $(\mathrm{n}=25)$, with no statistical

5 difference between the sexes $(p=0.371)$. Median NTBI in the patient group was $0.38 \mu \mathrm{M}$ (range:

$6 \quad 0.02-2.72 \mu \mathrm{M})$, slightly below that in healthy volunteers with statistical significance $(p=0.0144)($ Fig. $1 \mathrm{~A})$.

7 In the patient group, median NTBI was $0.39 \mu \mathrm{M}$ (range: $0.02-2.72$ ) in males and $0.38 \mu \mathrm{M}$ (range:

8 0.02-2.33) in females with no statistical difference observe between the groups. 2 patients in the patient

9 group showed elevated NTBI level; these patients had a transfusion history of receiving more than 10

10 units of packed red blood cells. Among the individual patient groups, median NTBI levels in ML

11 (median: $0.36 \mu \mathrm{M}$, range: 0.02-0.73 $\mu \mathrm{M}$ ) and MM (median: $0.38 \mu \mathrm{M}$, range: 0.23-0.73 $\mu \mathrm{M}$ ) showed

12 statistically significant decreases compared to that in healthy volunteers: $p=0.0273$ and $p=0.0265$,

13 respectively (Fig. 1B). No substantial within-day variation was observed in 3 healthy individuals (Fig. 2).

14 The relationship between NTBI and hemoglobin and biochemical markers such as AST, ALT,

15 serum creatinine, and albumin showed no statistically significant correlations in the patient group. A

16 slightly positive correlation was observed between sFe and NTBI (Fig. 3A), whereas no significant

17 correlation was observed between serum ferritin levels and NTBI (Fig. 3B). 
compared with CRP-positive and -negative groups. NTBI levels and sFe were significantly decreased in

3 inflammation (Fig. 4A, 4C). Conversely, median serum ferritin levels in the CRP-positive group were

4 significantly increased compared to that in the CRP-negative group (Fig. 4B).

\section{$5 \quad$ NTBI in iron-overloaded mice}

6 Serum NTBI levels were quantified in mice with and without peritoneal iron. NTBI levels were 0.11

$7 \pm 0.06,0.09 \pm 0.07$, and $0.57 \pm 0.37 \mu \mathrm{M}$ in the control, Fe $5 \mathrm{mg}$, and Fe $50 \mathrm{mg}$ groups respectively. A

8 statistically significant increase in NTBI levels were found in the Fe 50 mg group compared to the control

9 group (Fig. 5).

10 Changes in NTBI in rats after iron administration

11 To evaluate serum NTBI dynamics in iron loading, NTBI in rat serum was quantified after

12 intravenous iron administration. Average NTBI in the control rats was $0.16 \pm 0.04 \mu \mathrm{M}$, whereas NTBI

13 just 1 hour after iron injection was $2.78 \pm 0.62 \mu \mathrm{M}$. NTBI levels then decreased to $0.94 \pm 0.33 \mu \mathrm{M}$ and

$14 \quad 0.24 \pm 0.07 \mu \mathrm{M} 3$ and 6 hours respectively after iron injection (Fig. 6).

\section{NTBI in rats in inflammatory conditions}

16 To further confirm that NTBI is decreased in inflammation, rat experiments were performed.

17 Because basal NTBI levels are low in normal rats, iron with and without LPS were administered to rats

18 and the changes in NTBI levels observed. Three hours after oral iron administration, serum NTBI levels 
1 were found to rise significantly compared to the control group. On the other hand, the degree of NTBI

2 increase was suppressed in the rats administered with both iron and LPS (Fig. 7).

3 


\section{Discussion}

With our newly developed automated quantification system, we successfully quantified NTBI in

3 healthy volunteers and patients with various diseases, and found that NTBI is present even in the serum of

4 healthy persons (Fig. 1A). However in females, Tf was significantly less saturated than in males (35.6\%

5 in males and $22.9 \%$ in females: $\mathrm{p}=0.0052$ ); thus NTBI levels in females were expected to be lower, but no

6 statistical difference in NTBI levels were observed between the sexes. This is compatible with results

7 from a previous study using a non-metal HPLC measuring system [13], which indicates that NTBI may

8 be present in serum in constant levels, with some physiological function, however further investigation

9 will be necessary.

The correlation between NTBI and two widely-used iron metabolism biomarkers, sFe and serum

11 ferritin, showed a slight positive correlation between sFe and NTBI $(r=0.4579, \mathrm{p}<0.0001)$ (Fig. 3A); as

12 sFe levels reflect $\mathrm{Tf}$-bound iron, this finding indicates that NTBI levels vary when iron is in dynamic

13 equilibrium with Tf. However, some patients showed relatively high NTBI levels of $>0.5 \mu \mathrm{g} / \mathrm{dL}$, even

14 when sFe levels were moderate. sFe increases in parallel with $\mathrm{Tf}$ saturation, but soon reaches a plateau, as

15 sFe indicates iron bound to Tf, whose concentration is limited to serum. This limits the use of sFe to the

16 precise monitoring of iron toxicity, especially during iron-replete states. NTBI on the other hand can be

17 observed even when Tf is fully saturated; thus sFe and NTBI can be considered as biologically different

18 markers in iron metabolism. 
2 NTBI (Fig. 3B). However, we observed the interesting finding that NTBI levels decreased statistically,

3 whereas serum ferritin increased in inflammation in human serum (Fig. 4A, 4B). Further, NTBI levels

4 decreased in LPS-induced inflammation in iron-loaded rats (Fig. 7). In response to inflammation,

5 hepcidin expression is up-regulated, resulting in decreased iron absorption from the gut and blockade of

6 iron release from the reticuloendothelial system $[14,15]$. Thus suppressed serum iron may account for the

7 decreased NTBI levels in patients with inflammation. Moreover, although it is still not clear whether

8 NTBI contributes to the erythropoietic iron supply, the decrease in NTBI in inflammation may play a role

9 in the pathophysiology of the anemia of chronic disease. Despite serum ferritin being the most widely

10 used biomarker for total body iron stores, it is only useful in the absence of inflammation [16], hence the

11 unique in vivo characteristics of NTBI, as compared to serum ferritin, may help overcome the

12 disadvantage of serum ferritin, and could provide additional information regarding alterations in iron

13 metabolism even in inflammatory states.

14 NTBI was found to be present in our animal models of iron overload. Additionally, in both mice

15 peritoneally injected with iron and rats orally administered with iron, NTBI levels increased (Fig. 5, 6).

16 Further, serum NTBI rapidly increased and then decreased in rats intravenously injected with iron. These

17 observations indicate that NTBI may be useful not only as a marker for evaluating iron overload, but also

18 to precisely monitor dynamic changes of iron in the serum (Fig. 6). Although experimental iron 
1 administration rapidly produced NTBI in serum, it is important to note that this occurred in rats receiving

2 reasonably high doses of iron, and thus may not directly reflect what occurs at the physiologic level in

3 clinical patients receiving standard iron medication.

4 In the present study, we have demonstrated that compared to serum ferritin, NTBI is a unique

5 biomarker of iron metabolism. Finally, while the physiologic and clinical significance of NTBI remains to

6 be elucidated, we can conclude that our new automated method for measuring NTBI may be a powerful

7 tool for investigating the physiological and clinical importance of NTBI.

8 


\section{Conflict of Interest:}

2 Satoshi Ito, Katsuya Ikuta, Lynda Addo, Kotoe Shibusa, Yasumichi Toki, Mayumi Hatayama, Masayo

3 Yamamoto, Motohiro Shindo, Yutaka Kohgo, Mikihiro Fujiya (Division of Gastroenterology and

4 Hematology/Oncology, Department of Medicine, Asahikawa Medical University and Department of

5 Gastrointestinal Immunology and Regenerative Medicine, Asahikawa Medical University) received

6 research funding from Shino-Test Corporation; this study was also performed in collaboration with

7 Shino-Test Corporation. The above mentioned departments also received collaborative research funding

8 from Novartis Pharma K.K., Asahi Kasei Medical Co. Ltd., and Chugai Pharmaceutical Co. Ltd. for iron

9 metabolism research work. 
1. Kwiatkowski JL. Management of transfusional iron overload -differential properties and efficacy of iron chelating agents. J Blood Med. 2011;2:135-49.

2. Brissot P, Ropert M, Le Lan C, Loréal O. Non-transferrin bound iron: A key role in iron overload and iron toxicity. Biochim Biophys Acta. 2012;1820:403-10.

3. Taher AT, Musallam KM, Inati A. Iron overload. consequences, assessment, and monitoring. Hemoglobin. 2009;33 Suppl 1:S46-57.

4. Belotti A, Duca L, Borin L, et al. Non transferrin bound iron (NTBI) in acute leukemias throughout conventional intensive chemotherapy: Kinetics of its appearance and potential predictive role in infections complications. Leuk Res. 2015;39:88-91.

5. Prakash M, Upadhya S, Prabhu R. Serum non-transferrin bound iron in hemodialysis patients not receiving intravenous iron. Clin Chim Acta. 2005;360:194-8.

6. Malyszko J, Koc-Zorawska E, Levin-Iaina N, et al. Iron metabolism in hemodialysis patients - a story half told? Arch Med Sci. 2014;10:1117-22.

7. List AF, Baer MR, Steensma DP, et al. Deferasirox reduces serum ferritin and labile plasma iron in RBC Transfusion-dependent patients with myelodysplastic syndrome. J Clin Oncol. 2012;30:2134-9.

8. Cortelezzi A, Cattaneo C, Cristiani S, et al. Non-transferrin-bound iron in myelodysplastic syndromes: a marker of ineffective erythropoiesis? Hematol J. 2000;1:153-8.

9. Goto T, Ikuta K, Inamoto Y, et al. Hyperferritinemia after adult allogeneic hematopoetic cell transplantation: quantification of iron burden by determining non-transferrin-bound iron. Int J Hematol. 2013:97:125-134

10. Le Lan C, Loréal O, Cohen T, et al. Redox active plasma iron in C282Y/C282Y hemochromatosis. Blood. 2005;105:4527-31.

11. Breuer W, Hershko C, Cabantchik ZI. The importance of non-transferrin bound iron in disorders of iron metabolism. Transfus Sci 2000;23:185-92.

12. Ito S, Ikuta K, Kato D, et al. Non-transferrin-bound iron assay system utilizing a conventional automated analyzer. Clin Chim Acta. 2014;437:129-35.

13. Sasaki K, Ikuta K, Tanaka H, et al. Improved quantification for non-transferrin-bound iron measurement using high-performance liquid chromatography by reducing iron contamination. Mol

Med Rep. 2011;4:913-8.

14. Kroot JJ, Tjalsma H, Fleming RE, Swinkels DW. Hepcidin in Human Iron Disorders: Diagnostic Implications. Clin Chem. 2011;57:1650-69.

15. Sasaki Y, Shimonaka Y, Ikuta K, et al. Hepcidin production in response to iron is controlled by monocyte-derived humoral factors. Int J Hematol. 2014:99:12-20. 


\section{Figure legends}

$2 \quad$ Fig. 1

3 NTBI in healthy volunteers and patients. A: Median NTBI in patient group was

$4 \quad 0.38 \mu \mathrm{M}$ (range: $0.02-2.72 \mu \mathrm{M}$ ), with slight decrease in statistical significance

$5 \quad(p=0.0101)$ compared to that in healthy volunteers (median: $0.45 \mu \mathrm{M}$, range: $0.28-0.66 \mu \mathrm{M})$.

6 B: NTBI levels in individual disorders. NHL: non-Hodgkin lymphoma, AML: acute myeloid leukemia,

7 MDS: myelodysplastic syndrome, MM: multiple myeloma, MT: malignant tumors in digestive organs,

8 CML: chronic myeloid leukemia, MPN: myeloproliferative neoplasms, CD: Crohn's disease, Transplant:

9 post transplantation.

10

11 Fig. 2

12 The substantial within-day variation showing no variation in 3 healthy individuals.

13

$14 \quad$ Fig. 3

15 Relationship between serum iron markers and NTBI. A: sFe and NTBI showed slight positive correlation

$16(r=0.4579)$. B: No positive correlation between serum ferritin and NTBI $(r=0.2334)$.

$18 \quad$ Fig. 4 
1 A: Comparison between median NTBI in CRP-positive ( $>0.3 \mathrm{mg} / \mathrm{dL})$ and negative ( $\leqq 0.3 \mathrm{mg} / \mathrm{dL})$ groups;

2 NTBI in CRP-positive group was significantly decreased. B: Median serum ferritin levels were

3 significantly increased in CRP-positive group compared to CRP-negative group. C: Median sFe were

4 significantly decreased in the CRP-positive group compared to that in the CRP-negative group ( $\left.{ }^{*} p<0.05\right)$.

5

6

$7 \quad$ Fig. 5

8 Quantification of sFe (A) and NTBI (B) in mice with or without peritoneal iron. In the Fe 50 mg group,

9 which received $50 \mathrm{mg}$ of iron dextran, both sFe and NTBI was significantly increased compared to

10 control group $\left({ }^{*} p<0.05\right)$.

$12 \quad$ Fig. 6

13 Changes in NTBI levels in rats intravenously administered with iron. Rapid appearance of NTBI just 1

14 hour after iron injection and subsequent disappearance from serum after 6 hours. •; rats administered

15 with PBS, $\square$; rats administered with iron.

$17 \quad$ Fig. 7

18 NTBI in rats in inflammatory conditions. Compared to the control group, serum NTBI levels rise

19 significantly three hours after oral iron administration, whereas the degree of NTBI increase is suppressed

20 in rats administered with both iron and LPS. 


\section{Table1 Profiles of patients}

\begin{tabular}{|c|c|c|c|c|c|c|c|c|c|c|c|}
\hline & $\mathbf{n}$ & $\begin{array}{l}\text { Transfusion } \\
\text { history (unit) }\end{array}$ & WBC $(/ \mu \mathrm{L})$ & $\mathrm{Hb}(\mathrm{g} / \mathrm{dL})$ & Plt $\left(x 10^{3} / \mu \mathrm{L}\right)$ & $s F e(\mu \mathrm{g} / \mathrm{dL})$ & TIBC ( $\mu \mathrm{g} / \mathrm{dL})$ & $\begin{array}{l}\text { serum ferritin } \\
(\mathrm{ng} / \mathrm{mL})\end{array}$ & AST (U/L) & ALT (U/L) & CRP (mg/dL) \\
\hline NHL & 34 & $0(0-82)$ & $4,550(420-34,150)$ & $10.3(7.4-15.3)$ & $157(49-274)$ & $89(14-229)$ & 266 (139-394) & $196(30-2,568)$ & $24(9-216)$ & $23(7-81)$ & $0.15(0.10-9.19)$ \\
\hline AML & 11 & $22(0-52)$ & $2,500(330-6,440)$ & $8.4(7.5-15.6)$ & $130(7-437)$ & $162(34-250)$ & $278(208-467)$ & $723(148-2,817)$ & $25(7-81)$ & $26(6-119)$ & $0.22(0.10-5.09)$ \\
\hline MDS & 10 & $6(0-94)$ & $6,615(1,130-12,090)$ & $9.9(6.8-13.1)$ & $116(18-276)$ & $155(28-262)$ & $261(172-355)$ & $598(103-2,345)$ & $26(20-36)$ & $18(11-67)$ & $0.10(0.10-6.42)$ \\
\hline MM & 10 & $4(0-22)$ & $4,445(1,180-16,440)$ & $12.7(7.9-15.0)$ & $150(45-298)$ & $87(14-129)$ & $302(235-368)$ & $131(49-676)$ & $25(17-38)$ & $21(8-44)$ & $0.10(0.10-7.86)$ \\
\hline $\begin{array}{l}\text { Malignant tumors } \\
\text { in digestive organs }\end{array}$ & 15 & $0(0-2)$ & $3,910(980-5,910)$ & $11.1(6.7-14.4)$ & $150(31-422)$ & $71(13-138)$ & $276(176-443)$ & $124(7-548)$ & $28(13-95)$ & $20(6-130)$ & $0.15(0.10-4.83)$ \\
\hline CML & 7 & - & $115,650(2,850-237,610)$ & $12.0(9.0-14.1)$ & $211(46-394)$ & $108(85-356)$ & $335(245-375)$ & $139(10-1,017)$ & $22(15-47)$ & $19(12-61)$ & $0.14(0.10-2.78)$ \\
\hline MPN & 4 & $0(0-4)$ & $20,030(6,870-117,450)$ & $11.8(9.6-16.5)$ & $293(33-381)$ & $57(20-97)$ & $324(251-414)$ & $21(6-2,104)$ & $24(17-29)$ & $14(13-18)$ & $0.10(0.10-6.19)$ \\
\hline CD & 3 & - & $4,900(3,690-9,600)$ & $11.7(9.8-12.3)$ & $254(196-346)$ & 34 (21-72) & $312(297-368)$ & $31(11-206)$ & 18 (14-19) & $24(8-32)$ & $0.10(0.10-2.20)$ \\
\hline others & 22 & $0(0-100)$ & $6,025(1,490-50,660)$ & $11.6(6.8-19.7)$ & $190(61-319)$ & $89(8-266)$ & $270(174-416)$ & $361(2-4,000)$ & $25(11-128)$ & $25(7-196)$ & $0.10(0.10-12.23)$ \\
\hline
\end{tabular}

NHL: non-Hodgkin lymphoma, AML : acute myeloid leukemia, MDS : myelodysplastic syndrome, MM : multiple myeloma, CML : chronic myeloid leukemia, MPN : myeloproliferative neoplasms, CD : Crohn's disease, sFe : serum iron, TIBC : total iron binding capacity 
A

$$
\mu \mathrm{M}
$$

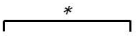

正

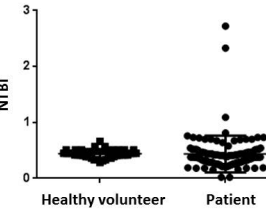

B

$$
\mu \mathrm{M}
$$

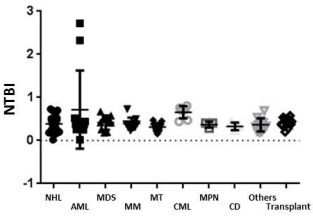




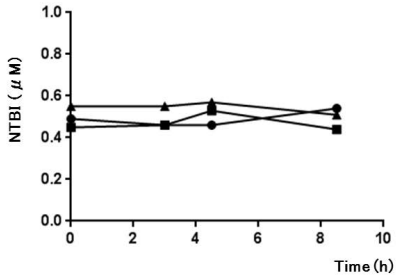


A

$\mu \mathrm{M}$

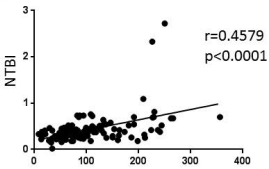

sFe
B

$\mu \mathrm{M}$

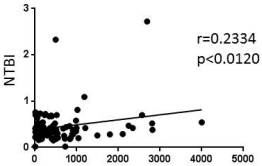

ferritin 


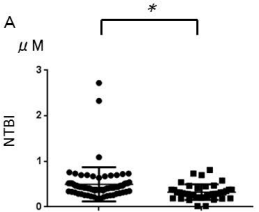

CRP-negative CRP-positive

C
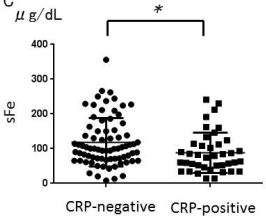
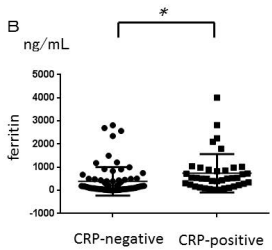
A

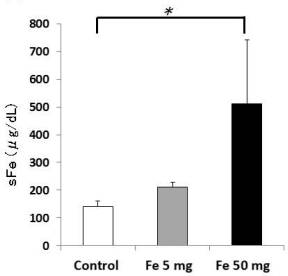

B

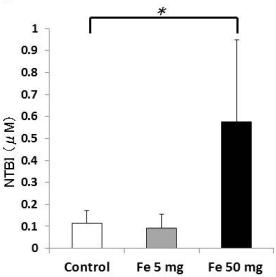




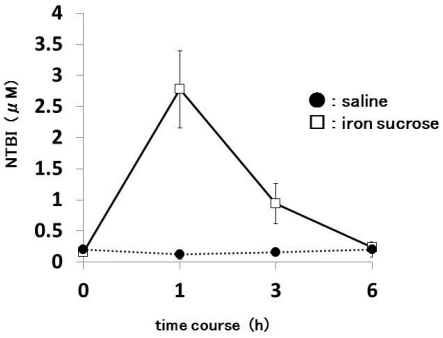




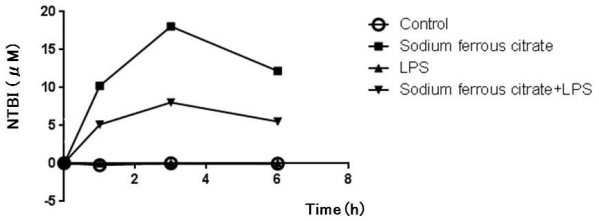

